\title{
THE PITTSBURGH SYMPOSIUM ON GROUP THEORY AND QUANTUM MECHANICS
}

In connection with the Annual Meeting of the Society at Pittsburgh, it was arranged to hold a symposium on Group theory and quantum mechanics under the joint auspices of the American Mathematical Society and the American Physical Society. The program was planned in co-operation with Professors H. P. Robertson and J. H. Van Vleck.

The symposium was held on Saturday afternoon, December 29, in the Union Room of the Carnegie Institute of Technology, and was followed by an interesting discussion; Professor Robertson presided. There was a large attendance of mathematicians and physicists. Abstracts* of the several addresses appear below.

I. Representations and ray-representations in quantum mechanics, by Professor John von Neumann.

It is known that any symmetry property of an intuitively described physical system finds its mathematical expression in the existence of a certain group under the operations of which the mechanical determining equations of the system are invariant. This group-theoretical principle of symmetry has been particularly suitable for group-theoretical methods. The mathematical theory, the application of which has in this connection led to many results in various fields of quantum physics, is the so-called theory of representations. As the states of the quantum mechanical system are really described, not by a uniquely defined wave-function, but by one which is only known-and has a physical meaning only-to within a constant factor of absolute value 1 , the discussion must be based not on the theory of representations proper, but on that of representations to within a constant factor of absolute value 1 . These have been called ray-representations. If the group of symmetry operations is a continuous (Lie) group, the representations may be discussed by considering the so-called infinitesimal representations of the group. These are characterized by certain commutation properties which, in the case of a rayrepresentation, will only be valid to within an additive constant multiple of unity. In some cases, these extra terms can be eliminated (transformed away). In some other cases they vanish automatically. There exist groups, however, for which neither is the case and the extra terms play an essential role. These possibilities are discussed for various main types of symmetry-groups.

* Because of illness, Professor von Neumann was unable to read the paper which he had prepared. It is nevertheless desirable to include the abstract of his work in this report for it furnishes a natural introduction to the other papers and the notions contained therein were referred to by the other speakers. 
II. Symmetry relations in various physical problems, by Professor E. P. Wigner.

Because of the linearity of the quantum mechanical equations, the operations of displacement and rotation in space-time must be represented by linear operators. These operators carry over the wave function of a state in a certain frame of reference into the wave function of the same state in another frame of reference. They form a representation of the group of possible coordinate transformations, which is the inhomogeneous Lorentz group. If these transformations are known, the equation of motion for the system can be derived from them, because they tell in particular how the state is changed by a shift of the time scale. Thus there is a unique correspondence between the possible Lorentz invariant equations of quantum mechanics, on the one hand, and the representations of the inhomogeneous Lorentz group by linear operators, on the other.

The gain in generality as compared with the usual tensor calculus consists in that no assumption concerning the field nature of the equations is made. Thus one must obtain in the present calculus all equations, even such (if they exist) in which the coordinate is quantized, for example.

The irreducible representations of the inhomogeneous Lorentz group were determined. There are, mathematically, three cases, according to whether $p_{t}^{2}-p_{x}^{2}-p_{y}^{2}-p_{z}^{2}$ is positive, zero, or negative, where the $p$ 's represent the infinitesimal displacement operators. The first case corresponds to a positive rest mass; in this case, it can be proved that all the representations may be interpreted as tensor equations. The same is true for the last case, which, however, has probably no significance. In the second case, which corresponds to photons, a new class of representations enters, which falls into the half-reducible class.

The detailed discussion will appear elsewhere in a joint paper with P. A. M. Dirac, who first perceived this problem.

III. Some applications of group theory to non-relativistic physical problems, by Professor J. H. Van Vleck.

Some groups encountered in non-relativistic quantum mechanics are the permutation group, the free rotation group, and crystallographic groups. The utility of the permutation group is greatly diminished by the fact that only a small fraction of the irreducible representations are compatible with the Pauli exclusion principle. A nice application of the free rotation group is to the calculation of the intensity distribution in the multiple structure of quadrupole lines, which are forbidden by the ordinary dipole selection rules, but are of importance in astrophysics. Two important applications of the crystallographic groups are to the theory of magnetism, and to the theory of the small vibrations of the nuclei about their equilibrium positions in polyatomic molecules. If the molecule has symmetry elements, then as E. P. Wigner and E. B. Wilson, Jr., have both shown, the theory of the characters and irreducible representations of the crystallographic group tells us how many normal vibrations should coincide in frequency, and which should be active in infra-red absorption or in the Raman effect. Also it permits a factorization of the secular equation which materially reduces the labor of calculating the fundamental frequencies. 


\section{Some applications of group theory to Dirac's relativistic theory, by Professor Gregory Breit.}

1. Dirac's relativistic theory introduces four matrices $\alpha_{\mu},(\mu=1,2,3,4)$, which satisfy the equation $\alpha_{\mu} \alpha_{\nu}+\alpha_{\nu} \alpha_{\mu}=2 \delta_{\mu \nu}$. According to a general theorem given by $\mathrm{P}$. Jordan and $\mathrm{E}$. Wigner by means of the theory of finite group representations there exists one and only one irreducible representation of these by means of finite matrices which is of interest for Dirac's equation. As shown by Pauli, this fact suffices for the proof of the relativistic invariance of Dirac's equation. The commutation relations of the $\alpha_{\mu}$ may be thus held responsible for the success of Dirac's equation as a relativistic description of the electron.

2. Using the matrices $\Gamma^{1}, \Gamma^{2}, \Gamma^{3}, \Gamma^{4}=\alpha_{4} \alpha_{1}, \alpha_{4} \alpha_{2}, \alpha_{4} \alpha_{3},-i \alpha_{4}$ and $x^{1}, x^{2}, x^{3}$, $x^{4}=x, y, z, i c t$ it is possible to find, for every Lorentz transformation $x^{\prime \nu}=b_{\mu}{ }^{\nu} x^{\mu}$ a matrix $\gamma$ such that $\gamma^{-1} \Gamma^{\nu} \gamma=b_{\mu}{ }^{\nu} \Gamma^{\mu}$. It was shown by von Neumann that these representations of the Lorentz group are capable of being represented, for pure space-time rotations, in two independent two-dimensional subspaces of the wave function components $\psi_{\mu}$. This is essentially due to the existence of a matrix $\Gamma$ with eigenwerte $+1,+1,-1,-1\left(\Gamma=\Gamma^{1} \Gamma^{2} \Gamma^{3} \Gamma^{4}\right)$ which commutes with all $\gamma$ which represent pure rotations. Taking linear combinations of the $\psi_{\mu}$ which correspond to the eigenaxes of $\Gamma$, one has two-dimensional representations of the proper Lorentz group. For transformations involving reflections $\Gamma, \gamma$ do not commute and a four-dimensional representation is necessary. The two-dimensional representations are now known as spinor representations.

Except for differences of notation the spinor treatment is already contained in Weyl's discussion of Dirac's equation where the isomorphism of the unimodular group of linear transformations of two variables with the group of Lorentz transformations was made use of and the difference between pure rotations and reflections was clearly set forth.

3. By methods of group theory or by the direct elegant approach of Dirac in his book one can discuss possible forms of relativistic interaction energy. Among the possible forms with four component equations only the Dirac and Pauli forms and their combinations are possible under the extended Lorentz group. For pure rotations other forms such as the one proposed by Rojansky are available. By the same methods one can discuss relativistically possible forms of proton, neutron, electron, neutrine interactions. One has the following possibilities without introducing derivatives of wave functions:

$$
\begin{gathered}
-F_{0}\left(\Psi^{*} \Psi\right)+F_{1}\left(\Psi^{*} \alpha_{1} \Psi\right)+F_{2}\left(\Psi^{*} \alpha_{2} \Psi\right)+F_{3}\left(\Psi^{*} \alpha_{3} \Psi\right) \\
-E_{0}\left(\Psi^{*} \rho_{1} \Psi\right)+E_{1}\left(\Psi^{*} \sigma_{1} \Psi\right)+E_{2}\left(\Psi^{*} \sigma_{2} \Psi\right)+E_{3}\left(\Psi^{*} \sigma_{3} \Psi\right) \\
F_{0}=\phi_{2} \psi_{1}-\phi_{1} \psi_{2}+\phi_{3} \psi_{4}-\phi_{4} \psi_{3}, \quad F_{1}=-\phi_{2} \psi_{4}+\phi_{4} \psi_{2}+\phi_{1} \psi_{3}-\phi_{3} \psi_{1}, \\
E_{0}=\phi_{4} \psi_{1}-\phi_{2} \psi_{3}-\phi_{3} \psi_{2}+\phi_{1} \psi_{4}, \quad E_{1}=\phi_{2} \psi_{2}-\phi_{2} \psi_{4}-\phi_{1} \psi_{1}+\phi_{3} \psi_{3},
\end{gathered}
$$

etc., with Dirac's original matrices as well as corresponding combinations with $-\left(\psi^{*} \phi\right), \psi^{*} \vec{\alpha} \psi$ and $-\left(\psi^{*} \rho_{1} \phi\right), \psi \vec{\sigma} \phi$. Here $\Psi$ refers to the heavy particle, $\phi$ and $\psi$ to the two types of light particles. The first form is Fermi's and the other forms are alternative possibilities.

J. R. KLINE, Associate Secretary 